\title{
Two Rare Cases of Monostotic Paget's Disease of Bone in Adult Female
}

\author{
Manohar TH*, Giriraj Harshavardhan JK and Gopinath Menon P \\ Department of Orthopaedic Surgery, Sri Ramachandra Institute of Higher Education and Research, India
}

*Corresponding author: Manohar TH, Department of OrthopaedicsSurgery, Sri Ramachandra Institute of Higher Education and Research, Porur, Chennai -600116, Tamil Nadu, India

\begin{abstract}
Introduction: Paget's disease of bone is a metabolic bone condition characterized by increased bone resorption by osteoclasts with simultaneous new bone formation. The result of which is bone appears expanded with both lytic and sclerotic areas. The bone eventually becomes weak and starts producing symptoms such as pain, difficulty in walking if it involves the weight bearing bones, pathological fracture and systemic symptoms related to increased bone turnover.

Case presentation: Here we are reporting two adult female cases of monostotic Paget's disease involving tibia. A 58-year-old female and a 47 year old female who presented with complaints of pain in the leg with history of fever. Blood investigations showed elevated alkaline phosphatase with normal serum calcium and phosphorous. X ray of tibia showed lytic and sclerotic areas. MRI showed features of Paget's disease of tibia. Biopsy of tibia confirmed the diagnosis to be monostotic Paget's disease of tibia. They were managed conservatively with oral bisphosphonates.

Conclusion: Monostotic Paget's disease even though is a rare disease it is encountered now and then in Indian population. It has to be differentiated from other lesions producing similar clinical picture such as secondary osteosarcoma, sclerosing osteomyelitis and metastasis. Early diagnosis and appropriate treatment will reduce pain, deformity and the need for surgical intervention with good symptomatic and radiological recovery.
\end{abstract}

Keywords: Paget's Disease; Monostotic Type; Tibia; Rare Condition; Indian Population

\section{Introduction}

Paget's disease of bone was first described by Sir James Paget as early as in the year 1877 [1]. This disease is also known as osteitis deformans. It can either involve a single bone (monostotic) or can involve many bones (polyostotic). This is a type of metabolic bone disease characterized by increased bone resorption and formation of new bone which is consistent with the presence of both lytic and sclerotic areas on radiology. The excessive remodelling makes the bone enlarged, weak and deformed. This disease commonly affects elderly white adults of age above 50 years. The prevalence of Paget's disease increases with age and is slightly more common in men. Paget's is rare under 25 years and unusual before 40 years of age [2].

\section{Case 1}

58-year-old female came with complaints of pain over the right knee for the past 2 weeks and difficulty in weight bearing over right lower limb. Pain was insidious in onset, aggravated in the night and relieved with rest and medications. No history of radiation of pain or history of pain elsewhere in the body. There was history of fever and there was no history of trauma. There was no history of loss of weight or loss of appetite. On examination there was no swelling, no scars or no sinuses, no visible pulsations or no dilated veins over right knee, there was no bowing. Warmth was present over the right proximal tibia, tenderness was present over right proximal tibia with broadening and thickening. The range of movements of knee joint was not affected and was not associated with any pain, there was no distal neurovascular deficits and distal pulses were well felt. Laboratory investigations showed normal calcium $(9 \mathrm{mg} / \mathrm{dl})$ and normal phosphorus $(3.7 \mathrm{mg} / \mathrm{dl})$. Serum alkaline phosphatase levels were elevated (148 IU/L). Plain x ray was done which showed sclerosis over right proximal tibia and lytic lesion over right tibial tuberosity with cortical thickening and widening (Figure 1,2). 


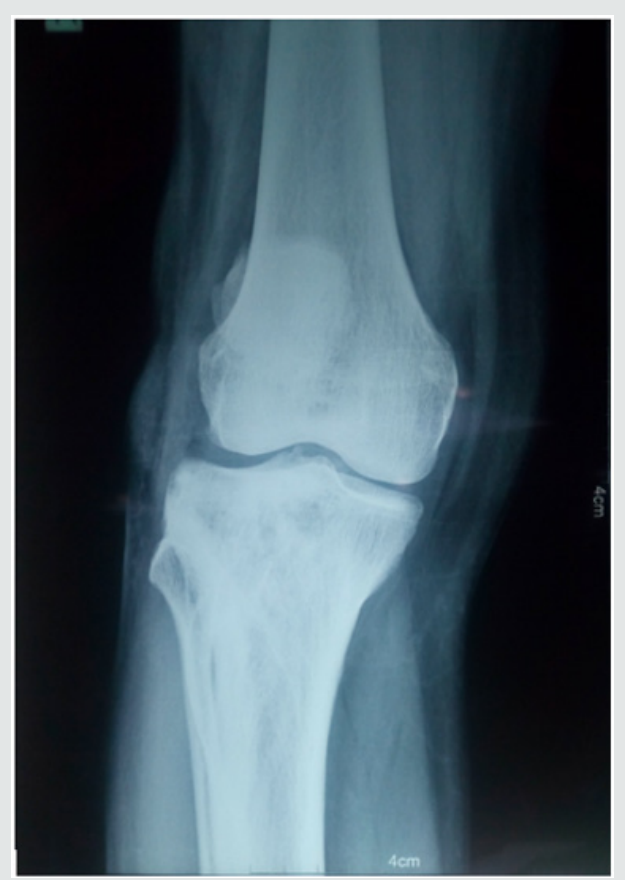

Figure 1: $\mathrm{X}$ ray of right proximal tibia antero-posterior view.

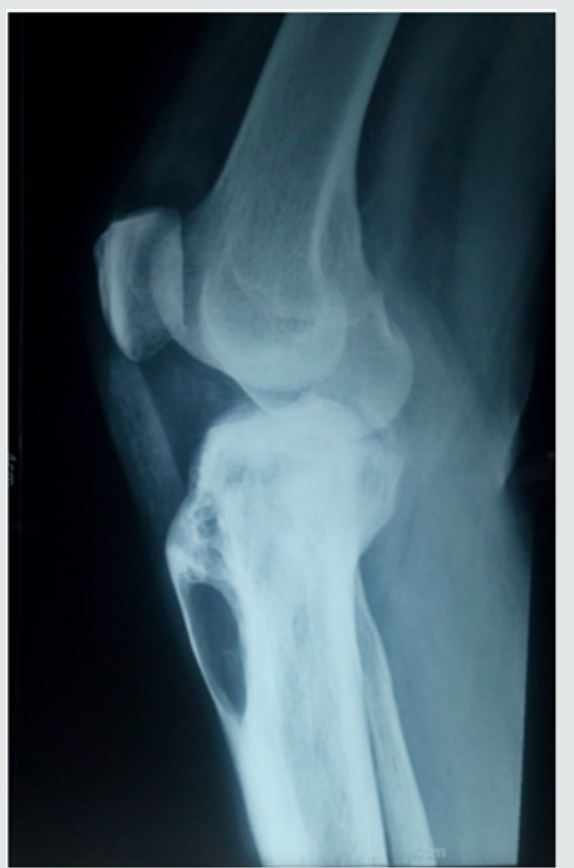

Figure 2: $\mathrm{X}$ ray of right proximal tibia lateral view.

We had differential diagnosis as Garre's sclerosing osteomyelitis of right proximal tibia, metastasis of right proximal tibia and secondary osteosarcoma. MRI of the right proximal tibia was done which showed diffuse cortical thickening of the involved tibia, heterogenous signal with normal fatty marrow, an intracortical cystic lesion measuring $4.2 \times 1.5 \times 2.3 \mathrm{~cm}$ noted just below the tibial tuberosity without any pathological fracture which was consistent with Paget's disease (Figure 3). CT guided biopsy was done from the lesion to confirm the diagnosis and the specimen was sent for histopathological study which came as monostotic Paget's disease of right tibia (Figure 4,5). She was treated with analgesics for pain and was started on oral bisphosphonates T. Alendronate $70 \mathrm{mg}$ weekly along with calcium supplementation. On follow up patient was symptomatically better and is mobilising well. 


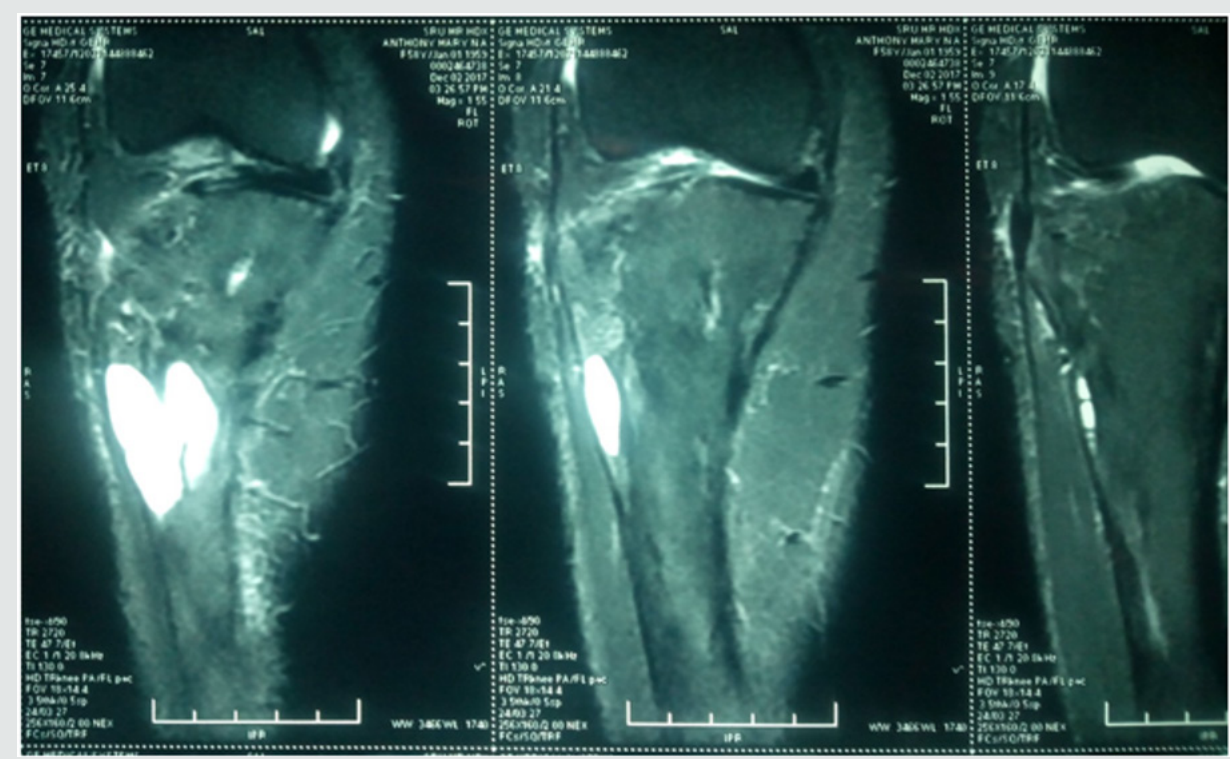

Figure 3: MRI right knee.

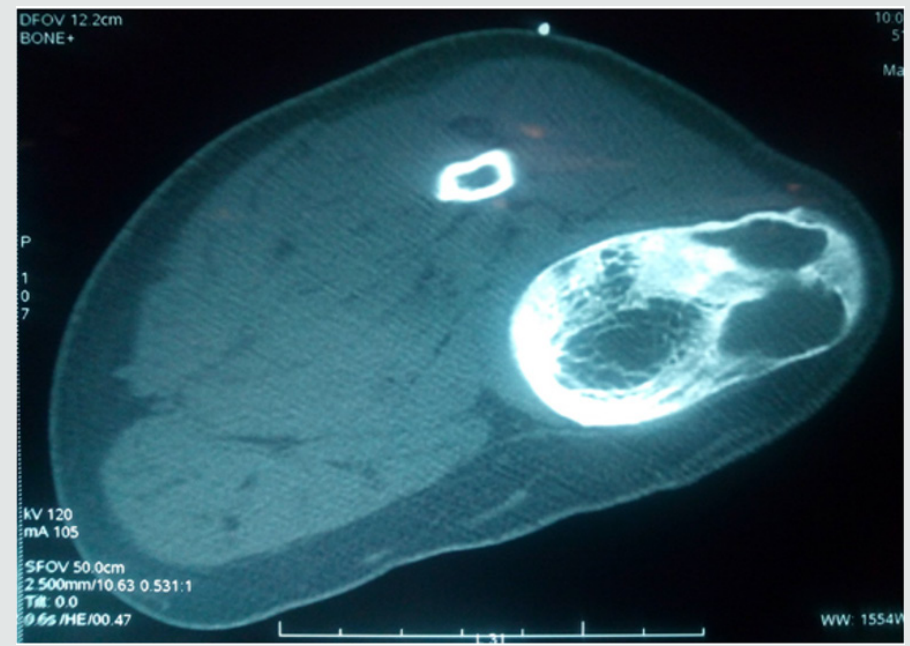

Figure 4: CT of right tibia.

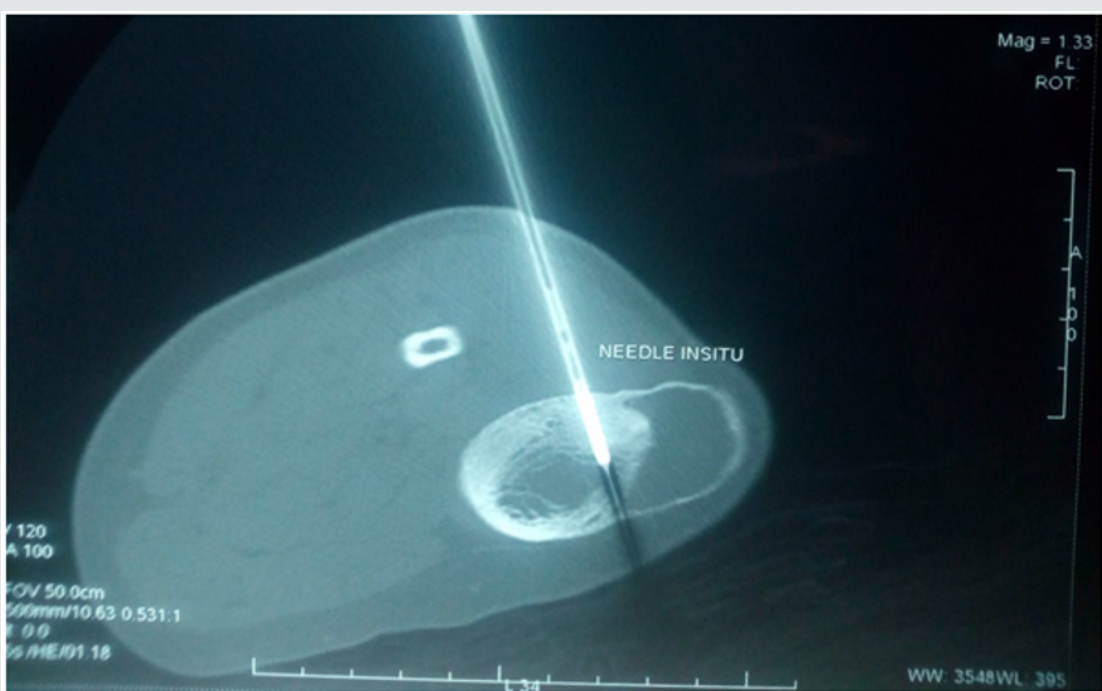

Figure 5: Needle insertion under CT guidance. 


\section{Case 2}

47-year-old female came with complaints of pain in the right leg and deformity of the right leg for one month. Pain is insidious in onset, aggravated on weight bearing, relieved on rest and medication. There was no radiation in pain, increased in night. Pain was associated with deformity and the deformity aggravated on weight bearing. There was no history of fever, no history of loss of weight or loss of appetite, no history of any other limb involvement and no history of trauma.On examination there was a Varus deformity of 10 degrees, there was no visible swelling, no dilated veins or no scars or no sinuses or any visible pulsations. There was warmth and tenderness diffusely over the right leg. Varus of the right leg of 10 degrees. Range of movements of knee and ankle were not affected and was not associate with any pain. There was no distal neurovascular deficits and distal pulses were well felt (Figure 6,7). Laboratory findings were calcium was normal
$(9.3 \mathrm{mg} / \mathrm{dl})$ and phosphorous was normal $(4.4 \mathrm{mg} / \mathrm{dl})$. Serum Alkaline phosphatase was elevated (173 IU/L ). ESR and CRP were normal. Plain $\mathrm{x}$ ray was done which showed lysis and sclerosis of entire right tibia with widening and thickening of cortex (Figure 8). We had differential diagnosis as Garre's sclerosing osteomyelitis of tibia, metastasis and secondary osteosarcoma of tibia. MRI right leg was done which showed involvement of entire tibia predominantly the proximal aspect including the articular cartilage with cystic areas, subcutaneous edema and thickened cortex with features of mixed phases of Paget's disease (Figure 9,10). Core needle biopsy of the right proximal tibia was done to confirm the diagnosis and the specimen was sent for histopathological study. The diagnosis was confirmed to be monostotic Paget's disease of right tibia. She was started on oral Alendronate $70 \mathrm{mg}$ once a day weekly along with calcium supplementation and analgesia for pain. Deformity correction was planned on a later date but the patient was symptomatically better and was mobilised so did not come.

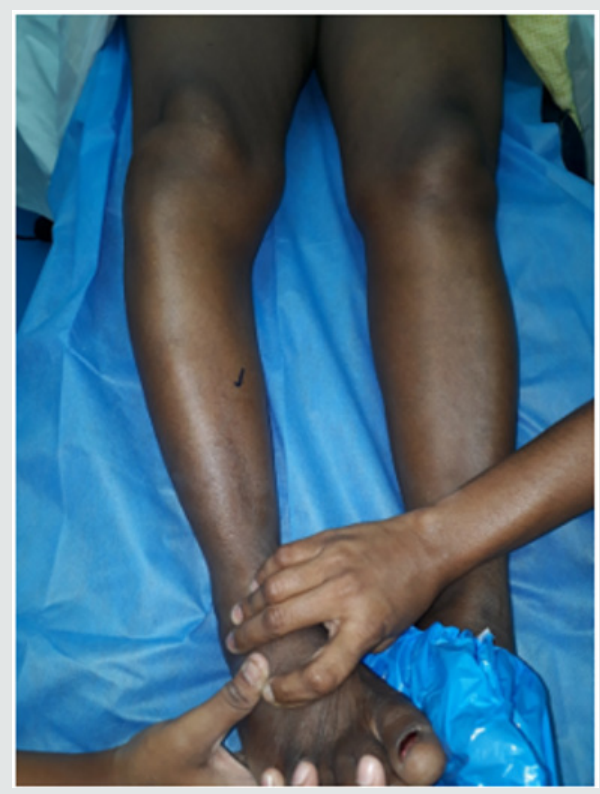

Figure 6: Clinical picture.

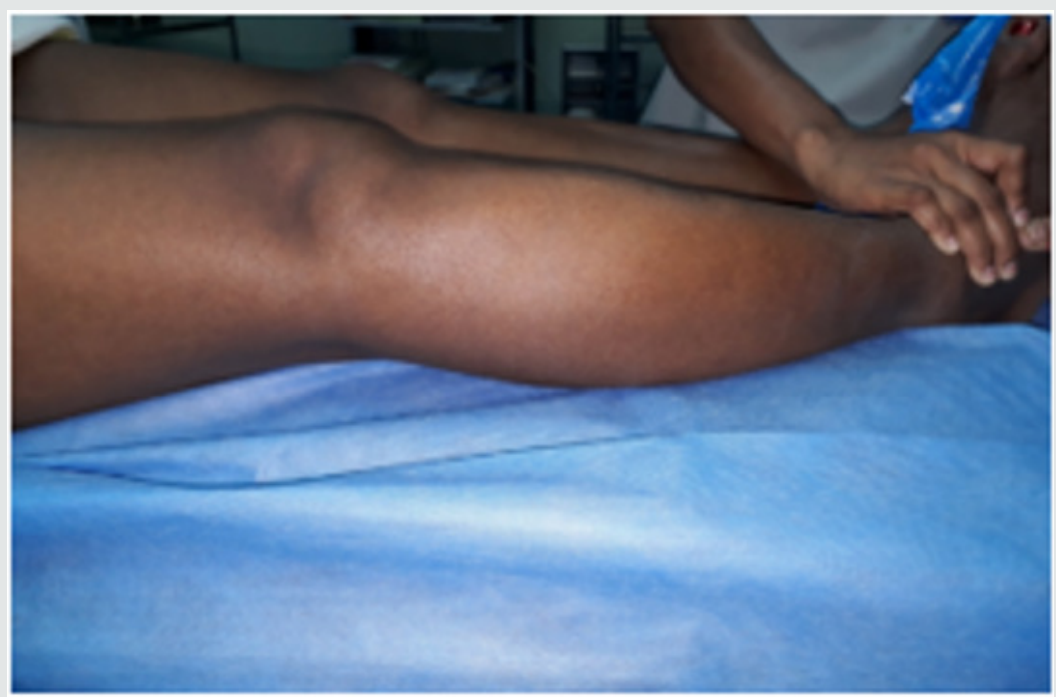

Figure 7: Clinical picture. 


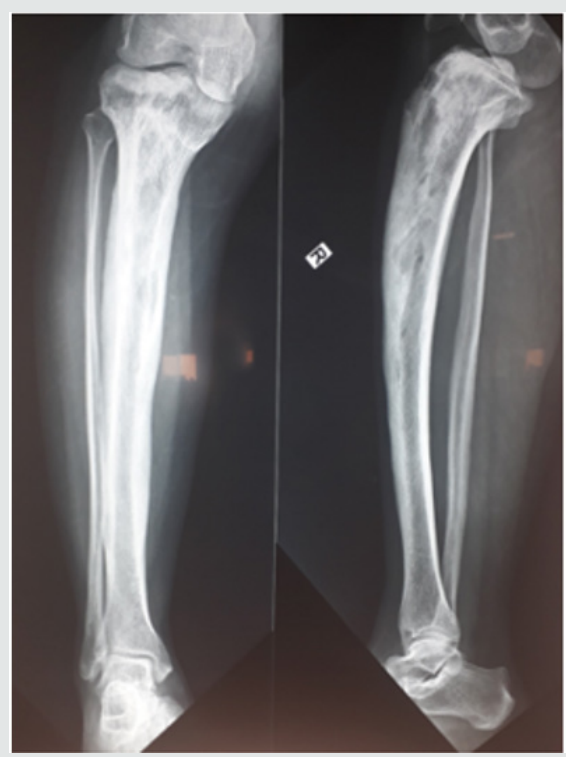

Figure 8: X ray of right leg antero-posterior and lateral view showing cortical widening with lysis and sclerosis predominantly involving proximal tibia and articular cartilage with Varus deformity.

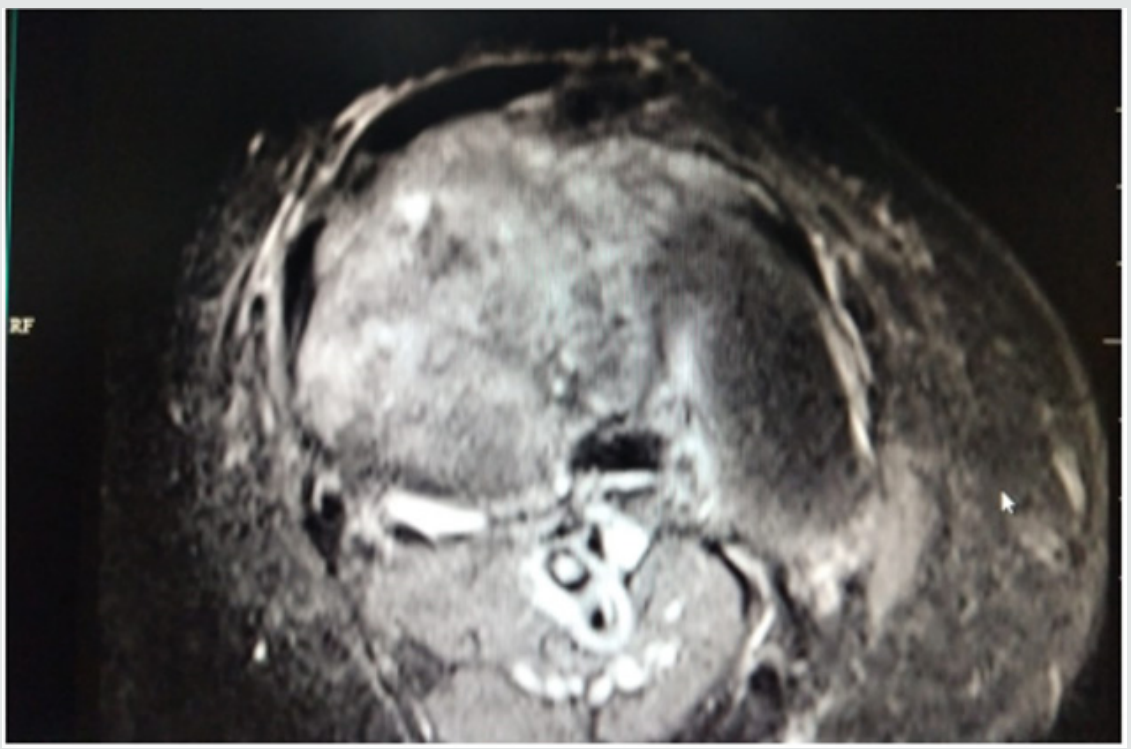

Figure 9: MRI of right knee showing cystic areas and edema inside the marrow and subcutaneous space.

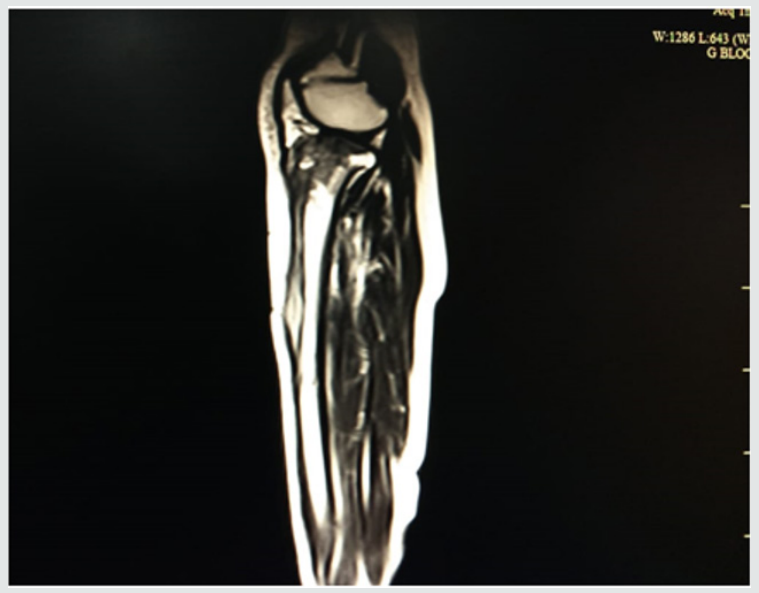

Figure 10: MRI of right knee. 


\section{Discussion}

Paget's disease of bone also known as osteitis deformans is a skeletal disease seen in elderly above 50 years of age characterized by increased bone resorption and increased bone turnover. It has a familial tendency of transmission common in first degree relatives of affected patients but it can also be caused by certain viral infections such as paramyxovirus or measles virus Paget's disease is most common in the United Kingdom and Western Europe but is also common in British immigrants to Australia, New Zealand, South Africa, and South America. The disease is uncommon in African blacks, Scandinavia, China, Japan, Southeast Asia, and the Indian subcontinent [3]. First-degree relatives of patients with Paget's disease have an increased risk particularly if the patient has an early age of diagnosis and the disease is extensive [4]. This suggests that there is familial trend and genetic factor plays an important role in Paget's disease. Rural life and animal contacts are associated with a greater risk of Paget's disease in [5,6], this suggests that animals may carry infectious agents causing the disease. The nuclear inclusion bodies in osteoclasts in Paget's disease resemble that of viruses [7]. but if viruses play a role as a causative agent for the disease remains unclear. Classical PDB is caused due to mutations in SQSTM1 that encodes for a protein known as p62 that plays an important role in osteoclast regulation. These mutations are only in $5-10 \%$ of sporadic cases and in $40-50 \%$ of familial cases [8]. Paget's disease is a chronic disease involving the skeletal system primarily. It is characterized by increased bone resorption by osteoclast followed by increased bone formation by osteoblasts. The osteoclasts are increased in number and size and may contain more than one nuclei. The nuclei may contain inclusion bodies that resemble viral particles [9].The initial pathology is because of increased resorption of bone by osteoclast due to abnormal activation of RANKL and increased expression of interleukin-6 ( IL-6) , the osteoblasts are also increased in number but they are morphologically normal suggesting that the main pathology is due to osteoclastic resorption of bone.

The accelerated resorption and turnover of bone causes the bone to be expanded and laying down of immature collagen fibres makes the bone structurally weak and the bone deforms In India Paget's disease is rare $[10,11]$.Many times the disease is diagnosed incidentally on routine blood or radiological investigations. In the musculoskeletal system the disease is characterised by pain over the involved bone, there is warmth due to increased vascularity, Deformity of the bone is present especially in weight bearing bones example bowing of femur or tibia, pathological fractures, osteoarthritis of joints adjacent to the involved bone. If the skull is involved there will be frontal bossing. If the spine is involved there will be back pain, radiating pain if there is canal stenosis or cord compression, in severe cases there can be paraplegia. The most commonly involved bones are spine, skull, pelvis, femur and tibia. Here one patient had pain, the other patient had pain and deformity with involvement of tibia in both the cases. Serum calcium, Serum phosphorous levels, Parathyroid hormone levels and Vit D levels [12] are usually normal unless the disease is extensive involving many areas. Bone specific alkaline phosphatase (BAP) seems to have the best diagnostic accuracy as a measure of bone formation, with a sensitivity of $84 \%$ and a specificity of $100 \%$ [13]. Both the patients who presented to us had normal serum phosphorous, normal serum calcium levels, normal parathyroid hormone and elevated alkaline phosphatase levels. Plain X ray of the involved site will show mixed areas of lytic and sclerotic lesions, bony enlargement, cortical thickening, increased trabeculae and bowing if weight bearing bones are involved. In the above cases we saw all findings in $\mathrm{x}$ rays.

Isotope bone scan shows increased uptake in affected areas. Here we did not do isotope bone scan instead we did MRI of the involved leg which showed features of Paget disease.

To confirm the diagnosis we did biopsy, computed tomography guided biopsy of tibia using trephine needle for one patient and core needle biopsy of tibia for the other patient and the specimen was sent for histopathological study. In both the cases it was reported as monostotic Paget disease consistent with our MRI findings.Our cases are unique in the sense both the patients were young female one in early 50's and the other in late 40's and both of them had primary involvement of only tibia without any other bone involvement such as pelvis, femur which are most common sites. Asymptomatic lesions do not need any treatment but regular follow up is necessary. In lesions producing symptoms or causing bowing of large weight bearing bones need treatment. The main goal of treatment is to prevent osteoclastic resorption of bone. Bisphosphonates or calcitonin can be used while bisphosphonates being gold standard of treatment for medical management of Paget's disease. Bisphosphonates such as etidronate, pamidronate, alendronate, risedronate and zoledronic acid [13]. While alendronate and risedronate are given orally whereas pamidronate and zoledronic acid are given intravenously. The potency of bisphosphonates in inhibiting osteoclastic bone resorption may be related to their ability to inhibit farnesyl diphosphate synthase [14]. Common side effects with oral bisphosphonates are gastrointestinal and oesophageal disturbances which is why it advised to take the drugs early in the morning. In those with gastrointestinal problems intravenous infusion of bisphosphonates can be given but there can be side effects such as transient bone pain, headache, myalgia and nausea. Response to treatment is observed by reduction in the level of serum alkaline phosphatase, improvement in bone pain and radiological improvement of the involved bone. Here once the diagnosis was confirmed by biopsy we started both the patients on oral alendronate $70 \mathrm{mg}$ weekly. Both the patients had symptomatic relief of bone pain and were mobilising well two months following the initiation of treatment.In a small number of patients surgery might be indicated if there is pathological fracture for fracture stabilisation, deformity of long bones which causes difficulty in walking or if the patient feels is cosmetically unacceptable in the form of corrective osteotomy, Paget's disease is known to cause secondary osteoarthritis hence in selected cases total joint arthroplasty maybe necessary [15]. Paget's disease is a highly vascular condition hence surgery should be done after adequate treatment of the disease to reduce blood loss. 


\section{Conclusion}

Monostotic Paget's disease even though is a rare disease it is encountered now and then in Indian population. It has to be differentiated from other lesions producing similar clinical picture such as secondary osteosarcoma, sclerosing osteomyelitis and metastasis. Early diagnosis and appropriate treatment will reduce pain, deformity and the need for surgical intervention with good symptomatic and radiological recovery.

\section{Consent}

The patients have given their consent for the case report to be published.

\section{Competing Interest}

The authors declare that the interpretation of data or presentation of information is not influenced by any personal or financial relationship with other people or organisations.

\section{References}

1. Paget J (1877) On a Form of Chronic Inflammation of Bones (Osteitis Deformans). Med Chir Trans 60(1): 37-64.

2. Siris E, Roodman GD (2008) Paget's disease of bone In: Rosen CJ, Compston JE, Lian JB (Eds.), Primer on the metabolic bone diseases and disorders of mineral metabolism, $7^{\text {th }}$ (edn), American Society for Bone and Mineral Research, USA, pp. 335-334.

3. Altman RD (2002) Epidemiology of Paget's disease of bone. Clin Rev Bone Miner Metab 1: 99-102.

4. Siris E (1991) Indications for medical treatment of Paget's disease of bone In: Singer FR, Wallach S, (Eds.), Paget's disease of bone: clinical assessment, present and future therapy, Elsevier, New York, USA.
5. Gennari L, Merlotti D, Martini G, Nuti RJ (2009) Paget's disease of bone in Italy. Bone Miner Res 21(2): 14-21.

6. López Abente G, Morales Piga A, Elena Ibáñez A, Rey Rey JS, Corres González J (1997) Cattle, pets, and Paget's disease of bone. Epidemiology 8(3): 247-251.

7. Mills BG, Singer FR (2014) Nuclear inclusions in Paget's disease of bone. Science 194(4261): 201-202

8. Robin S, Shivangi G, Shipra S, Rajat P, Saumyakanta M, et al. (2014) An Insight into Paget's Disease of Bone. Niger J Surg 20(1): 9-15.

9. Reddy SV, Singer FR, Mallette L, Roodman GD (1996) Detection of measles virus nucleocapsid transcripts in circulating blood cells from patients with Paget disease. Bone Miner Res 11(11): 1602-1607.

10. Sridhar GR (1994) Paget's disease in India: Is it truly rare? Natl Med J India 7(2): 101.

11. Motilal BG, Mayilavahanan N, Sriram V, Soundarapandian S, Shanmugasundaram TK (1992) Paget's disease (osteitis deformans). Ind J Orthop 16: 130-133.

12. Devlin RD, Retallack RW, Fenton AJ, Grill V, Gutteridge DH, et al. (1994) Long term evaluation of 1,25-dihydroxyvitamin D after short term intravenous administration of pamidronate in Paget's disease of bone. J Bone Miner Res 9(1): 81-85.

13. Licata AA (2005) Discovery, clinical development, and therapeutic uses of bisphosphonates. Ann Pharmacother 39(4): 668-677.

14. Dunford JE, Thompson K, Coxon FP, Luckman SP, Hahn FM, Poulter CD, et al. (2001) Structure-activity relationships for inhibition of farnesyl diphosphate synthase in vitro and inhibition of bone resorption in vivo by nitrogen-containing bisphosphonates. J Pharmacol Exp Ther 296(2): 235-242.

15. Parvizi J, Klein GR, Sim FH (2006) Surgical management of Paget's disease of bone. J Bone Miner Res 21(2): 75-82.

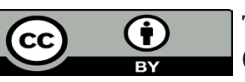

This work is licensed under Creative Commons Attribution 4.0 License

To Submit Your Article Click Here: Submit Article

DOI: $10.32474 /$ OSMOAJ.2020.03.000170

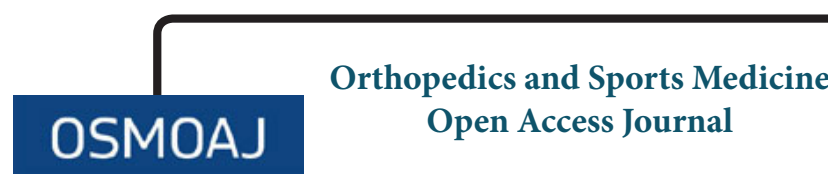

Assets of Publishing with us

- Global archiving of articles

- Immediate, unrestricted online access

- Rigorous Peer Review Process

- Authors Retain Copyrights

- Unique DOI for all articles 